\title{
Functional Age Predicted by Electronic Short Physical Performance Battery Can Detect Frailty Status in Older Adults
}

This article was published in the following Dove Press journal: Clinical Interventions in Aging

\author{
Hee-Won Jung $\mathbb{D}^{\prime}$ \\ Taeyang Jin' \\ ji Yeon Baek (iD) \\ Seongjun Yoon ${ }^{2}$ \\ Eunju Lee (D) \\ Jack M Guralnik ${ }^{3}$ \\ II-Young Jang $\mathbb{D}^{\prime}$ \\ 'Division of Geriatrics, Department of \\ Internal Medicine, Asan Medical Center, \\ Seoul, Republic of Korea; ${ }^{2}$ Dyphi \\ Research Institute, Dyphi Inc., Daejeon, \\ Republic of Korea; ${ }^{3}$ Division of \\ Gerontology, Department of \\ Epidemiology and Public Health, \\ University of Maryland School of \\ Medicine, Baltimore, MD, USA
}

Purpose: The importance of evaluating frailty status of older adults in clinical practice has been gaining attention with cumulative evidence showing its relevance in clinical outcomes and decision-making. We aimed to develop and validate whether the functional age predicted by an electronic continuous short physical performance battery (eSPPB) could predict frailty status.

Patients and Methods: We reviewed medical records of outpatients ( $\mathrm{N}=834)$ of Asan Medical Center, aged 51-95 years. We used the eSPPB data of 717 patients as a development cohort, and that of 117 patients, who also underwent comprehensive geriatric assessments, as a validation cohort. Frailty index was calculated by counting deficits of 45 geriatric items including comorbidities, daily functions, mobility, mood, and cognition. For functional age, we used balance score (0-4), gait speed ( $\mathrm{m} / \mathrm{s})$, and stand-up time (s) measured 5 times in the chair rise test.

Results: From the development cohort, we established a functional age using the formula $(83.61-1.98 *$ [balance score] $-5.21 *$ [gait speed] $+0.23 *$ [stand-up time] $)$, by multivariate linear regression analysis with chronological age as a dependent variable $\left(\mathrm{R}^{2}=0.233\right)$. In the validation cohort, the functional age positively correlated with frailty index $(\mathrm{p}<0.001)$. C-statistics classifying frailty (defined as frailty index $\geq 0.25)$ was higher $(p<0.001)$ with functional age (0.912) than that with chronological age (0.637). A cut-off functional age of $\geq 77.2$ years maximized Youden's J when screening for frailty, with sensitivity of $94.4 \%$ and specificity of $80.8 \%$.

Conclusion: A newly developed functional age predictor using eSPPB parameters can predict the frailty status as defined by the deficit accumulation method and may serve as a physical biomarker of human aging.

Keywords: frailty, biomarker, physical performance, diagnosis

\section{Introduction}

Clinical and research interests in identifying frailty in older adults have been ever increasing globally. ${ }^{1}$ Frailty is a common geriatric condition that is defined as a state of decreased physiological reserve and increased vulnerability to possible stressors. ${ }^{1}$ Several researchers have shown that it is associated with adverse health outcomes such as lower quality of life, ${ }^{2}$ institutionalization, ${ }^{3}$ and mortality. ${ }^{3}$ Furthermore, frailty status has been regarded as a risk predictor in specialized medical treatments such as cancer chemotherapy, and other medical therapies or surgical procedures. ${ }^{4,5}$ With researchers suggesting that adverse outcomes of frailty
Correspondence: II-Young Jang

Division of Geriatrics, Department of Internal Medicine, Asan Medical Center,

University of Ulsan College of Medicine, 88

Olympic-ro 43-gil, Songpa-gu, Seoul 05505,

Republic of Korea

Tel +82-2-30l0-1658

Email onezero2@gmail.com 
and frailty status per se can be amenable to adequately designed intervention schemes, ${ }^{6,7}$ detecting frailty in older adults in clinical practice is crucial. ${ }^{8}$

Currently, identifying frailty status has not been widely adopted in clinical practices except in geriatrics, even in developed countries with a high rate of population aging. There are various instruments to assess frailty, including screening questionnaires, ${ }^{9,10}$ operational criteria for phenotype, ${ }^{3}$ and indices counting deficits that are associated with human aging. ${ }^{11,12}$ However, assessments for frailty status that can be quickly performed with objective, functional parameters that are less prone to possible human errors are still lacking. ${ }^{1,13}$ Therefore, in specialized medical and surgical practices, constrained time and human resources may preclude widespread, routine assessments for frailty in older patients even with its clinical importance.

Although not originally aimed at detecting the frailty spectrum, components of the short physical performance battery (SPPB), ${ }^{14}$ which is a functional test evaluating standing balance, walking speed, and chair rise test, assess overlapping parameters with frailty phenotype. With these characteristics, SPPB score is predictably associated with frailty status and geriatric functional parameters. ${ }^{9,15,16}$ Based on this observation, we hypothesized that using sensor-based continuous parameters of standing balance, walking speed, and chair rise test from the newly developed electronic SPPB (eSPPB), ${ }^{15}$ functional age could be calculated to correlate with the frailty index obtained from the deficit accumulation model that has been deemed as a measure of biological age in studies. ${ }^{17}$ The eSPPB can be easily performed by less-experienced examiners in an objective manner with the help of computer-based instructions and automatic measurement using sensors. Hence, this hypothetical functional age from eSPPB may overcome the current problems in adopting frailty assessments in clinical practice.

Therefore, we aimed to establish an equation for functional age from a development cohort using the three parameters of sensor-based eSPPB. Furthermore, we aimed to validate the newly developed functional age from the validation cohort using the comprehensive geriatric assessment (CGA)-based frailty index in geriatric clinic outpatients at a tertiary academic hospital.

\section{Patients and Methods}

\section{Study Design and Population}

In this retrospective cross-sectional study, we used medical records of 834 patients who visited the geriatric outpatient clinic of Asan Medical Center from February 2019 to January 2020 and underwent eSPPB for their functional evaluation. In performing eSPPB, patients with apparent life expectancy less than 1 year due to malignancy and those with symptomatic heart failure or end-stage renal disease, patients not able to walk without assistance, and those with cognitive dysfunction who could not perform eSPPB according to instructions were excluded for the measurement of eSPPB, while communitydwelling and ambulatory (with or without walking aid) patients were considered for the test. In these 834 records of patients who performed eSPPB, records of 717 patients served as a development cohort (Figure S1), to establish an equation for functional age from eSPPB. Because of the previously known population-based correlation between chronological age and frailty index, ${ }^{12}$ we used chronological age as the dependent variable for the functional age in this population. However, CGA-based frailty index was not available in this population. We used medical records of 117 other patients (Figure S1), who had eSPPB data and also had undergone CGA, as the validation cohort. The study protocol was reviewed and approved by the Institutional Review Board of Asan Medical Center (S2018-1483-0001), complied the ethical rules for human experimentation that stated in the Declaration of Helsinki, ${ }^{18}$ and informed consent for this analysis was waived, regarding retrospective nature of the study, and potential risk on patients from the analysis was less likely. In the analysis, confidentiality of patients' health information was maintained, and analysis was performed using anonymized dataset.

\section{Measurement of Electronic Short Physical Performance Battery}

To measure the physical parameters of eSPPB, we used sensors and software (Dyphi, Daejeon, Korea) developed to measure standing balance, walking speed, and chair rise test as previously reported. ${ }^{15}$ An array with 16 load cells detecting the two-dimensional location and weight distribution of each foot was used for the standing balance test. The standing balance was measured in the side-by-side, semi-tandem, and tandem stances. The participants were asked to maintain the posture for up to $10 \mathrm{~s}$. For walking speed, the patients were asked to walk at their usual speed, which was measured with a previously validated onedimensional light detection and ranging (LiDAR) sensor that recorded the distance between the sensor and 
participants once every $10 \mathrm{~ms} .{ }^{19}$ For the chair rise test, patients were asked to stand up and sit down 5 times as fast as possible with arms folded on the chest. The time taken to complete the test was measured with two sensors, a load cell embedded chair measuring the weights of sitting participant every $10 \mathrm{~ms}$, and a small chip LiDAR range sensor measuring the distance between the buttocks of the patient and the chair. Algorithm of the computer software connected to the sensors automatically produced scores for components according to previously published cut-off points, to produce standing balance score $(0-4)$, walking speed score $(0-4)$, chair rise test score $(0-4)$, and SPPB total score $(0-12) .{ }^{14}$

\section{Comprehensive Geriatric Assessment and Frailty Index}

We used the frailty index from CGA as our main study outcome variable. CGA was performed by experienced nurses in the geriatric outpatient clinic, in patients included in validation cohort. Presence of comorbidities was assessed by reviewing medical records and interviewing patients. In the medication review, status of polypharmacy was defined as taking $\geq 5$ different medications per day. Mobility was assessed by self-reported measures using three mobility items by Rosow and Breslau, ${ }^{20}$ and five items of basic physical activities by Nagi. ${ }^{21}$ Any fall history in the past 1 year was recorded. Significant weight loss was defined as $\geq 4.5 \mathrm{~kg}$ reduction in body weight in the last 1 year. For cognitive function, the Korean version of Mini-Mental Status Examination was used. ${ }^{22}$ Activities of daily living (ADL) functions of dressing, washing, bathing, eating, moving, and using bathroom were recorded. Instrumental activities on daily living (IADL) functions of taking transportation, using phones, buying groceries, managing medications, managing finances, preparing foods, doing basic household chores, and washing clothes were recorded. Patients with $\geq 1$ impaired items were considered to have disabilities in ADL or IADL functions.

For quantitative evaluation of frailty burden in participants of the validation cohort, we built a frailty index counting accumulations of deficits associated with human aging, based on the widely accepted procedures using CGA parameters (Table S1) ${ }^{11,23}$ We included parameters entailing underlying diseases, physical and cognitive impairments, psychosocial risk factors, geriatric syndromes, and disability. The specific components of the frailty index in this study are listed in Table S1. The frailty index was calculated as the cumulative deficit of the 45 items ranging from 0 to 1 , where a higher score indicated a greater frailty burden. Frailty index of $\geq 0.25$ represented frailty, in accordance with a previous study. ${ }^{11}$

\section{Statistical Analysis}

To establish a conceptual functional age from eSPPB, we used multivariable linear regression analysis with original standing balance score, walking speed $(\mathrm{m} / \mathrm{s})$, and time (s) to finish the chair rise test as independent variables, and the chronological age as a dependent variable. $T$-test was used to compare age and physical performance between the development and validation cohorts. Mann-Whitney test and $\mathrm{X}^{2}$ test or Fischer's exact test were used to compare demographic and geriatric parameters according to the calculated functional age in the validation cohort. Linear regression analysis was performed to assess correlation between the functional age and frailty index in the validation cohort. Receiver operating characteristic (ROC) analysis was used to evaluate the classifying performance of chronological age and functional age with respect to the frailty index, and C-statistics from these ROC analyses were compared. Two-sided $p$-values $<0.05$ were considered statistically significant. Statistical analyses were performed using Stata 16.0 (StataCorp, College Station, TX, USA).

\section{Results}

\section{Establishment of a Conceptual Functional Age from Development Cohort}

The development cohort $(\mathrm{n}=717)$ comprised of 398 (55.5\%) females, and the mean age was 73.2 years (standard deviation [SD] 9.2, range 51-95). Mean SPPB total score was 10.1 (SD 2.6), and the distributions of SPPB total score and component specific scores are shown in Figure 1. A multivariable linear regression model produced the following equation for conceptual functional age with chronological age as a dependent variable $\left(\mathrm{R}^{2}=\right.$ $0.233, p<0.001$; Table 1 and Figure 2A):

Functional age $=83.61-1.98 *($ balance score, point $)-$ $5.21 *($ gait speed, $\mathrm{m} / \mathrm{s})+0.23 *($ stand-up time, $\mathrm{s})$

\section{Associations of Functional Age and Chronological Age with Frailty Status in Validation Cohort}

The validation cohort $(\mathrm{n}=117)$ included $59(50.4 \%)$ males, and the mean age was 77.2 years (SD 7.0). 


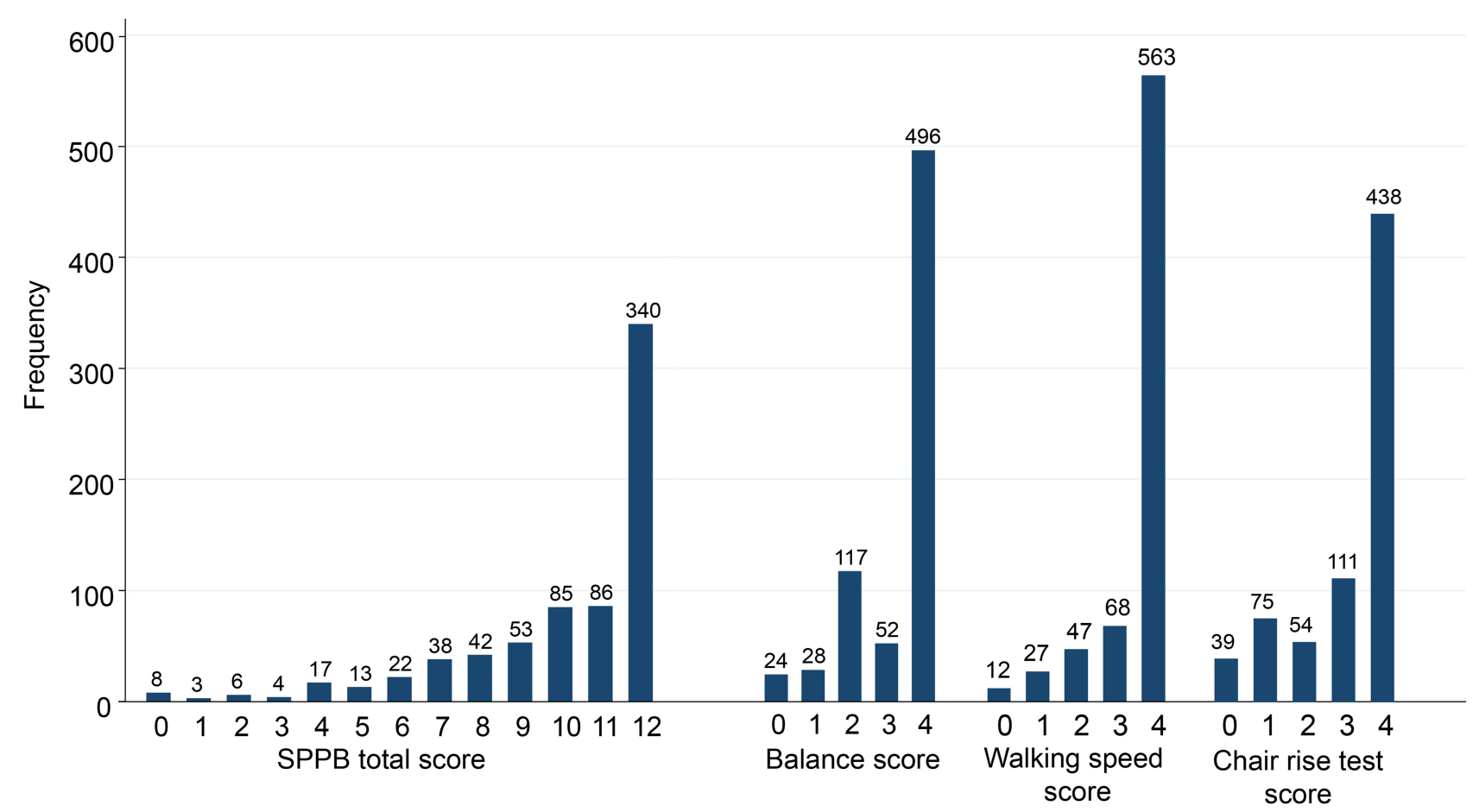

Figure I Distributions of short physical performance battery (SPPB) total score and balance score, walking speed score, chair rise score in development cohort ( $=7$ I7).

Baseline characteristics of the validation cohort are shown in Table 2, with population divided into two groups by the median value (74.2 years) of functional age of this cohort. Mean SPPB total score was 9.6 (SD 2.5). Patients in the validation cohort were older $(p<0.001)$ and had worse SPPB total score $(p=0.041)$ when compared to patients in the development cohort. In this population, mean frailty index was 0.14 (SD 0.12), and 18 (15.4\%) had frailty index of $\geq 0.25$ and were considered frail. The functional age from SPPB parameters positively correlated with the frailty index (Standardized beta $[\mathrm{B}]=0.66, \mathrm{R}^{2}=0.432, \mathrm{p}<$ 0.001 ; Figure $2 \mathrm{~B})$ as did the chronological age $(\mathrm{B}=0.34$, $\left.\mathrm{R}^{2}=0.118, \mathrm{p}<0.001\right)$. In the ROC analysis (Figure 3 ), C-statistics classifying frailty was higher $(p<0.001)$ for functional age (0.912) than for chronological age (0.637). A cut-off functional age of $\geq 77.2$ years maximized Youden's $\mathrm{J}$ in classifying frailty, with sensitivity of $94.4 \%$ and specificity of $80.8 \%$.

\section{Discussion}

This study showed that a conceptual functional age model based on the three physical parameters of eSPPB, which was developed to track chronological age, correlated with the frailty index from CGA in an independent population. The present study was the first to adopt recently developed sensors and software for eSPPB in a real-world clinical setting. This revealed that sensor-based continuous parameters of gait speed and chair rise test could be incorporated into a model to capture the spectrum of human aging. Also, as ROC analysis showed in the validation population, functional age from physical parameters was superior in classifying frailty than chronological age, supporting the concept that trajectory of aging and frailty are heterogenous among individuals.

Previously, numerous studies attempted to disentangle the biological mechanism and nature of frailty and human aging. ${ }^{24}$ In large human populations, association between

Table I Linear Regression Analysis Predicting Chronological Age From the Parameters of Short Physical Performance Battery in Development Cohort $(n=717)$

\begin{tabular}{|l|l|l|l|}
\hline & Coefficient (B) & 95\% Confidence Interval & p-value \\
\hline Balance score (increasing by I point) & -1.98 & -2.56 to -1.40 & $<0.00$ I \\
Walking speed (increasing by I m/s) & -5.21 & -6.60 to -3.82 & $<0.00$ I \\
Stand-up time (increasing by I s) & 0.23 & 0.12 to 0.33 & $<0.00$ I \\
\hline
\end{tabular}


A

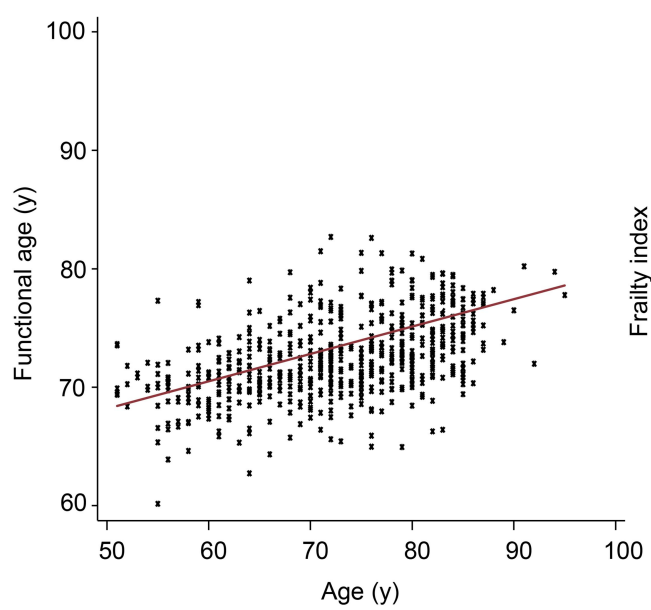

B

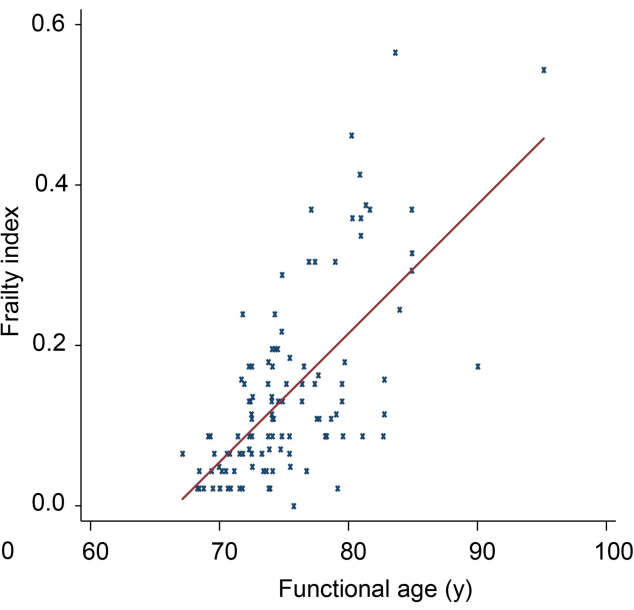

Figure 2 Scatterplot and fitted line by linear regression analysis showing (A) functional age and chronological age in development cohort $(n=7 / 7)$ and $(\mathbf{B})$ functional age and frailty index in validation cohort $(n=117)$.

frailty index and chronological age have been reported. ${ }^{12}$ Subsequent studies showed that frailty is associated with endocrinological dysregulation, chronic inflammation, and vascular damage. A recent proteomics study of human population supported these associations. ${ }^{25-27}$ Moreover, studies have suggested that frailty phenotype can be a consequence of cellular senescence, possibly mediated by senescence-associated secretory phenotype, and chronic inflammation, and this mechanism might be reversed in pre-clinical models. ${ }^{28,29}$

While many studies have focused on discovering molecular biomarkers of frailty that eventually might be translated to possible novel mode of action in treating human aging, we attempted to track frailty using physical, macroscopically measured dynamic parameters that can be rapidly acquired in a non-invasive manner. As Zampino et al suggested in a recent review, ${ }^{25}$ we aimed to measure the generalized phenotype of frailty in a spectrum manner rather than by disease-specific measures, which can predict clinical outcomes and be sensitive to interventions. By combining physical performance parameters of previously known abilities to predict outcomes and sensitivities to interventions, ${ }^{7,30,31}$ we could even predict frailty by deficit accumulation that includes chronic diseases as well as mobility parameters.

Table 2 Clinical Characteristics of Patients Included in the Validation Cohort, with Higher Functional Age and Lower Functional Age Group Defined by the Median Value of Functional Age (74.2 Years)

\begin{tabular}{|c|c|c|c|c|c|}
\hline & $\begin{array}{l}\text { Lower Functional Age ( }<74.2 \text { years), } \\
N=59\end{array}$ & & $\begin{array}{l}\text { Higher Functional Age ( } \geq 74.2 \text { years), } \\
N=58\end{array}$ & & $p$-value ${ }^{a}$ \\
\hline Chronological age & 74.2 & (7.2) & 80.3 & (5.3) & $<0.001$ \\
\hline Sex (male) & 27 & $(45.8 \%)$ & 32 & $(55.2 \%)$ & 0.309 \\
\hline Comorbidities & & & & & \\
\hline Hypertension & 25 & $(43.9 \%)$ & 39 & $(68.4 \%)$ & 0.008 \\
\hline Diabetes & 13 & $(22.8 \%)$ & 16 & $(28.1 \%)$ & 0.519 \\
\hline Depression & 12 & $(21.1 \%)$ & 16 & $(28.1 \%)$ & 0.514 \\
\hline Dementia & 3 & $(5.3 \%)$ & 8 & $(14.0 \%)$ & $0.203^{b}$ \\
\hline Frailty index & 0.08 & $(0.05)$ & 0.20 & $(0.13)$ & $<0.001$ \\
\hline Polypharmacy & 26 & $(45.6 \%)$ & 46 & $(80.7 \%)$ & $<0.001$ \\
\hline Fall history in I year & 5 & $(8.8 \%)$ & 19 & $(33.3 \%)$ & $0.002^{\mathrm{b}}$ \\
\hline ADL dependency & 3 & $(5.1 \%)$ & 17 & $(29.3 \%)$ & $<0.001^{\mathrm{b}}$ \\
\hline IADL dependency & 11 & $(18.6 \%)$ & 33 & $(56.9 \%)$ & $<0.001$ \\
\hline
\end{tabular}

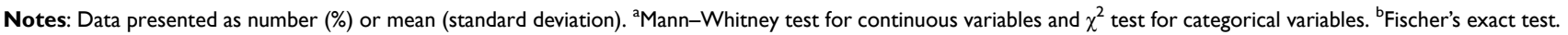
Abbreviations: ADL, activity of daily livings; IADL, instrumental activities of daily living. 

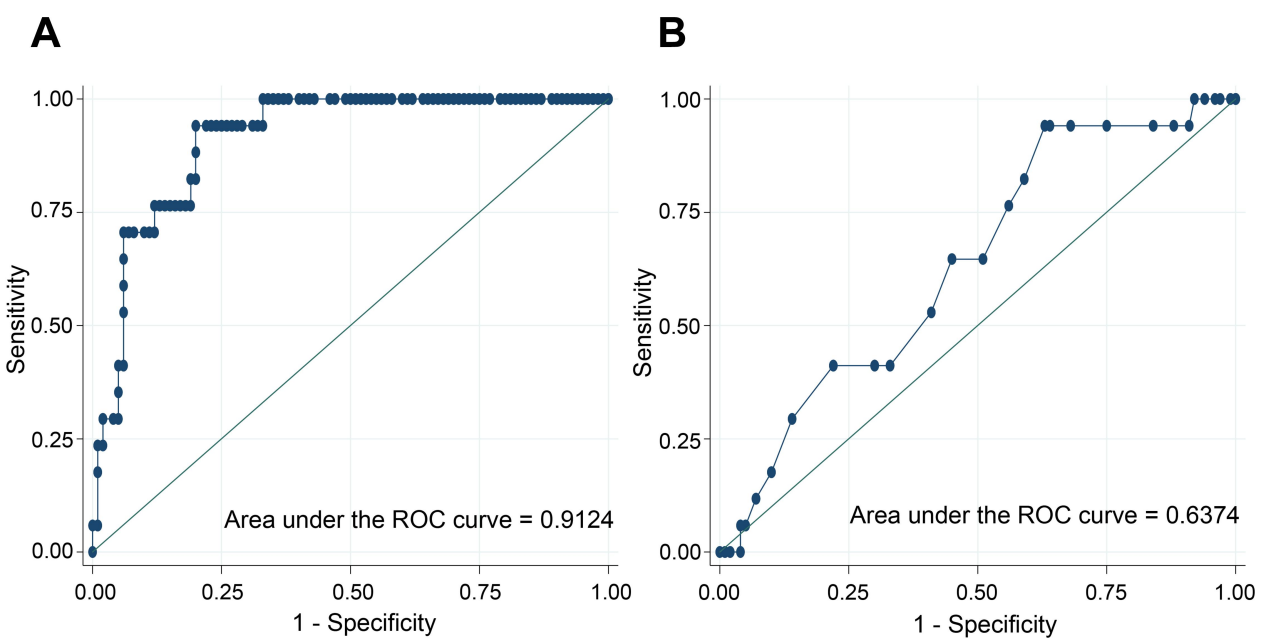

Figure 3 Receiver of characteristic (ROC) curves showing diagnostic performance of (A) functional age and (B) chronological age to detect frail status by frailty index in validation cohort $(n=117)$.

Even though the present study simply used the three parameters of balance score, continuous gait speed, and chair rise test time of eSPPB, elaborate algorithms using sensor derived parameters with accumulating clinical data may reveal values of previously lesser-known parameters that can be observed in physical performance tests. For example, initial acceleration of walking, walking speed variability, and other gait parameters including step width and length could be acquired and analyzed in a single walking test. With these advances, dynamic ranges and resolutions of physical parameter portfolio can be further improved in future studies.

Our findings on physical parameters and frailty index may have implications on developing schemes to measure frailty in model organisms. ${ }^{24,32}$ Previously, in rodent studies, biochemical or histological measures with survival analysis were commonly used to assess effects of agents that could delay aging phenotype or remove senescent cells. ${ }^{28}$ With intrinsic drawbacks in reliably measuring physical performance of rodents in behavioral experiments, physical biomarkers have been mostly regarded as supplementary measures in contrast to human trial designs that prioritize improvements in physical performance. ${ }^{33}$ However, with advances in sensor technologies, human physical biomarkers might be translated to animal models to close the current gaps between pre-clinical and clinical studies on frailty and aging.

Despite this study being the first to adopt novel sensors measuring physical performance in assessments of frailty in real world geriatrics clinic, it had several limitations. Since the study was based on outpatients of a tertiary hospital, the new equation for functional age from the validation cohort of this study may not be generalizable to other older populations. Although functional age was validated in an independent population that had undergone CGA, the size of the validation population was substantially smaller than the development population. Additionally, the validation population was older and had worse physical performance as compared to the development population, which may have produced biased results in the study. Further validation in a larger and balanced population with CGA is warranted.

\section{Conclusion}

In conclusion, functional age developed to correlate maximally with the chronological age using the three parameters of eSPPB could predict frailty index by CGA in geriatric outpatients. Functional age, calculated from eSPPB that can be performed in a relatively short time in a non-invasive manner, may serve as a physical biomarker of human aging.

\section{Acknowledgments}

This research was supported by a grant from the Korea Health Technology R\&D project through the Korea Health Industry Development Institute (KHIDI) funded by the Ministry of Health \& Welfare, Republic of Korea (grant no. HI18C2383), and a grant (grant no. 2018IF0143) from the Asan Institute for Life Science, Asan Medical Center, Seoul, Republic of Korea. There was no role of sponsor in any parts of this study including the design, methods, recruitment, data collections, analysis and preparation of paper. 


\section{Disclosure}

Hee-Won Jung and Seongjun Yoon cofounded Dyphi Inc, a startup company on sensor technology. Seongjun Yoon is a current employee of Dyphi Inc . The aforementioned authors report no other potential conflicts of interest in this work. The other authors do not have any conflicts of interest to report for this work.

\section{References}

1. Hoogendijk EO, Afilalo J, Ensrud KE, et al. Frailty: implications for clinical practice and public health. Lancet. 2019;394 (10206):1365-1375. doi:10.1016/S0140-6736(19)31786-6

2. Jung H-W, Jang I-Y, Lee YS, et al. Prevalence of Frailty and Aging-Related Health Conditions in Older Koreans in Rural Communities: a Cross-Sectional Analysis of the Aging Study of Pyeongchang Rural Area. J Korean Med Sci. 2016;31(3):345-352. doi: $10.3346 / \mathrm{jkms} .2016 .31 .3 .345$

3. Fried LP, Tangen CM, Walston J, et al. Frailty in older adults: evidence for a phenotype. J Gerontol a Biol Sci Med Sci. 2001;56 (3):M146-156. doi:10.1093/gerona/56.3.M146

4. Kim DH, Afilalo J, Shi SM, et al. Evaluation of changes in functional status in the year after aortic valve replacement. JAMA Intern Med. 2019;179(3):383-391.

5. Ruiz J, Miller AA, Tooze JA, et al. Frailty assessment predicts toxicity during first cycle chemotherapy for advanced lung cancer regardless of chronologic age. J Geriatr Oncol. 2018.

6. Dent E, Martin FC, Bergman H, et al. Management of frailty: opportunities, challenges, and future directions. Lancet. 2019;394 (10206):1376-1386.

7. Jang I-Y, Jung H-W, Park H, et al. A multicomponent frailty intervention for socioeconomically vulnerable older adults: a designed-delay study. Clin Interv Aging. 2018;13:1799-1814.

8. Jung H, Visualizing W. Domains of Comprehensive Geriatric Assessments to Grasp Frailty Spectrum in Older Adults with a Radar Chart. Ann Geriatric Med Res. 2020;24(1):55-56.

9. Morley JE, Malmstrom TK. Miller DK: A simple frailty questionnaire (FRAIL) predicts outcomes in middle aged African Americans. $J$ Nutr Health Aging. 2012;16(7):601-608.

10. Jung H-W, Yoo H-J, Park S-Y, et al. The Korean version of the FRAIL scale: clinical feasibility and validity of assessing the frailty status of Korean elderly. Korean J Intern Med. 2016;31(3):594-600. doi: $10.3904 / \mathrm{kjim} .2014 .331$

11. Rockwood K, et al. A global clinical measure of fitness and frailty in elderly people. CMAJ. 2005;173(5):489-495. doi:10.1503/ cmaj.050051

12. Mitnitski AB, Graham JE, Mogilner AJ, et al. Frailty, fitness and late-life mortality in relation to chronological and biological age. BMC Geriatrics. 2002;2(1):1. doi:10.1186/1471-2318-2-1

13. High KP, Zieman S, Gurwitz J, et al. Use of Functional Assessment to Define Therapeutic Goals and Treatment. J Am Geriatr Soc. 2019;67(9):1782-1790. doi:10.1111/jgs.15975

14. Guralnik JM, Simonsick EM, Ferrucci L, et al. A short physical performance battery assessing lower extremity function: association with self-reported disability and prediction of mortality and nursing home admission. J Gerontol. 1994;49(2):M85-94. doi:10.1093/geronj/49.2.M85
15. Jung H-W, Roh H, Cho Y, et al. Validation of a Multi-Sensor-Based Kiosk for Short Physical Performance Battery. J Am Geriatr Soc. 2019;67(12):2605-2609. doi:10.1111/jgs.16135

16. Jung H-W, Kim S-W, Ahn S, et al. Prevalence and outcomes of frailty in korean elderly population: comparisons of a multidimensional frailty index with two phenotype models. PLoS One. 2014;9(2): e87958. doi:10.1371/journal.pone.0087958

17. Song XW, Mitnitski A, Rockwood K. Rockwood K: prevalence and 10-Year Outcomes of Frailty in Older Adults in Relation to Deficit Accumulation. J Am Geriatr Soc. 2010;58(4):681-687. doi:10.1111/ j.1532-5415.2010.02764.x

18. World Medical A. World Medical Association Declaration of Helsinki. Ethical principles for medical research involving human subjects. Bull World Health Organ. 2001;79(4):373-374.

19. Jung H-W, Roh H-C, Kim S, et al. Cross-Comparisons of Gait Speeds by Automatic Sensors and a Stopwatch to Provide Converting Formula Between Measuring Modalities. Ann Geriatric Med Res. 2019;23(2):71-76. doi:10.4235/agmr.19.0016

20. Rosow I, Breslau N. Breslau N: A Guttman Health Scale for the Aged. J Gerontol. 1966;21(4):556-559. doi:10.1093/geronj/21.4.556

21. Nagi SZ. An epidemiology of disability among adults in the United States. The Milbank Memorial Fund Quarterly. Health and Society. 1976;54(4):439-467. doi:10.2307/3349677

22. Kang Y, Na DL. Hahn S: A validity study on the Korean Mini-Mental State Examination (K-MMSE) in dementia patients. J Korean Neurol Assoc. 1997;15(2):300-308.

23. Searle SD, Mitnitski A, Gahbauer EA, et al. A standard procedure for creating a frailty index. BMC Geriatrics. 2008;8(1):24. doi:10.1186/ 1471-2318-8-24

24. Kane AE, Sinclair DA. Frailty biomarkers in humans and rodents: current approaches and future advances. Mech Ageing Dev. 2019;180:117-128. doi:10.1016/j.mad.2019.03.007

25. Zampino M, Ferrucci L, Semba RD. Semba RD: biomarkers in the path from cellular senescence to frailty. Exp Gerontol. 2020;129:110750. doi:10.1016/j.exger.2019.110750

26. Sathyan S, Ayers E, Gao T, et al. Plasma proteomic profile of frailty. e. 2014. 13193.

27. Jung H-W, Kim S-W, Yoon S-J, et al. Associations between frailty, retinal microvascular changes, and cerebral white matter abnormalities in Korean older adults. $J$ Am Geriatr Soc. 2014;62 (11):2209-2210. doi:10.1111/jgs.13114

28. Xu M, Tchkonia $\mathrm{T}$, Ding $\mathrm{H}$, et al. JAK inhibition alleviates the cellular senescence-associated secretory phenotype and frailty in old age. $J$ Nat. 2015;112(46):E6301-E6310.

29. Baker DJ, Wijshake T, Tchkonia T, et al. Clearance of p16Ink4a-positive senescent cells delays ageing-associated disorders. Nature. 2011;479(7372):232. doi:10.1038/nature10600

30. Studenski S, et al. Gait speed and survival in older adults. JAMA. 2011;305(1):50-58. doi:10.1001/jama.2010.1923

31. Pavasini R, Guralnik J, Brown JC, et al. Short Physical Performance Battery and all-cause mortality: systematic review and meta-analysis. BMC Med. 2016;14(1):215.

32. Whitehead JC, Hildebrand BA, Sun M, et al. A clinical frailty index in aging mice: comparisons with frailty index data in humans. J Gerontol a Biol Sci Med Sci. 2014;69(6):621-632.

33. Reginster J-Y, Beaudart C, Al-Daghri N, et al. Update on the ESCEO recommendation for the conduct of clinical trials for drugs aiming at the treatment of sarcopenia in older adults. Aging Clin Exp Res. 2020;1-15. 


\section{Publish your work in this journal}

Clinical Interventions in Aging is an international, peer-reviewed journal focusing on evidence-based reports on the value or lack thereof of treatments intended to prevent or delay the onset of maladaptive correlates of aging in human beings. This journal is indexed on PubMed Central, MedLine, CAS, Scopus and the Elsevier
Bibliographic databases. The manuscript management system is completely online and includes a very quick and fair peer-review system, which is all easy to use. Visit http://www.dovepress.com/ testimonials.php to read real quotes from published authors. 